\title{
Effect of family-centered care interventions on motor and neurobehavior development of very preterm infants: a protocol for systematic review
}

Manasa Kolibylu Raghupathy ${ }^{1}$, Bhamini Krishna Rao ${ }^{1 *}$ D, Shubha R. Nayak', Alicia J. Spittle ${ }^{2}$ and Shradha S. Parsekar ${ }^{3}$

\begin{abstract}
Background: Globally, very preterm birth is a health concern leading to various developmental difficulties such as poor motor and/or cognitive function. For infants born very preterm, family-centered care (FCC) might promote developmental skills over the time in an appropriate enriched environment. The purpose of this study is to systematically review and assess the evidence of FCC interventions on the motor and neurobehavioral development in very preterm infants. Additionally, this review aims to determine the factors that might affect infant development.

Methods: Systematic review will be carried out by including (a) quasi-randomized controlled trials and randomized controlled trials (b) of very preterm born infants (born $<32$ weeks of gestation), and their primary caregivers will be included in the review (c) who received FCC-based interventions such as collaborative interaction between a healthcare professional and a parent, home program, home visits, and parent education, and (d) measure motor and neurobehavioral function. Electronic databases such as Scopus, PubMed, Embase, Cochrane Library, Web of Science, CINAHL, and PsycINFO will be searched using database-specific terms. Additionally, searches will be carried out in ProQuest, and references of included studies will be searched. Two review authors, independently, will conduct the screening, data extraction, and critical appraisal of included studies. If possible, a meta-analysis will be undertaken to assess the effect of the FCC on the motor and neurobehavior of premature infants.
\end{abstract}

Conclusion: The review will provide insights regarding the effect of the FCC on preterm infants. This systematic review will guide the clinicians on the feasibility of practicing FCC that might support and promote the integration of parents into various rehabilitation settings.

(Continued on next page)

\footnotetext{
*Correspondence: bhamini.kr@manipal.edu

'Department of Physiotherapy, Manipal College of Health Professions, Manipal Academy of Higher Education, Manipal 576104, India

Full list of author information is available at the end of the article
}

(C) The Author(s). 2021 Open Access This article is licensed under a Creative Commons Attribution 4.0 International License, which permits use, sharing, adaptation, distribution and reproduction in any medium or format, as long as you give appropriate credit to the original author(s) and the source, provide a link to the Creative Commons licence, and indicate if changes were made. The images or other third party material in this article are included in the article's Creative Commons licence, unless indicated otherwise in a credit line to the material. If material is not included in the article's Creative Commons licence and your intended use is not permitted by statutory regulation or exceeds the permitted use, you will need to obtain permission directly from the copyright holder. To view a copy of this licence, visit http://creativecommons.org/licenses/by/4.0/ The Creative Commons Public Domain Dedication waiver (http://creativecommons.org/publicdomain/zero/1.0/) applies to the data made available in this article, unless otherwise stated in a credit line to the data. 
(Continued from previous page)

Systematic review registration: Protocol has been registered in PROSPERO on August 26, 2020.

Keywords: Family-centered care, Motor development, Neurobehavior development, Very preterm infants, Systematic review

\section{Background}

\section{Description of the condition}

Infants born between 28 and 32 weeks of gestation are considered "very preterm" infants [1]. Globally, approximately 5 to $18 \%$ ( 15 million) of neonates are born preterm of all the live births [2]. The rate of very preterm born vary throughout the world, and in the USA, about $1.6 \%$ of live births are born as very preterm [3]. However, there is dearth of data on proportion of neonates born very preterm in other countries. These babies are at high risk of impaired cognitive, sensory, motor, emotional, language, and behavioral development when compared to full-term infants [4]. Early brain injury and impaired brain development are reasons for the aforementioned problems [5].

In the first year of life, biological, environmental, and social factors influence the development of early motor and neurobehavior development. Motor development encompasses the quality of movement, developmental milestones, motor skills, visual-spatial, visual-motor integration, balance, and coordination [6]. Neurobehavioral development occurs with efficient responses to the sensory (tactile, auditory, visual, olfactory) stimulation and autonomic nervous system, organization of state (calm, excited, irritable), and self-regulation (hand to mouth responses), language, attention, socio-emotional development, and executive function [7].

Very preterm infants are usually admitted in the neonatal intensive care units (NICU) to provide special care and management. In the NICU, very preterm infants face many stressful situations such as excessive sound, bright light, painful medical applications, and lack of parental contact, which they would not have experienced in utero [8]. Excessive sensory load on tactile, olfactory, gustatory, visual, and auditory systems during this critical period of brain development impairs baby's physiological responses and may impact negatively on their neuromotor and behavioral development. Therefore, the relationship between preterm birth and the aforementioned chain of events might affect the development of motor skills and neurobehavior in the later span of life [9].

Family and home-based environments are considered to have the greatest and permanent effect on an infant's growth within ecological system, even when taking into consideration of certain other aspects such as education and socioeconomic status of the parents [10]. Birth of a very preterm infant affects the mental well-being of the parents, leading to distress and anxiety, and alters the sense of bonding and parenting skills to care [11]. A review conducted to see the effects of discharge communication practices in pediatric emergency care reported parental education as an early intervention strategy that might provide a significant opportunity for improving parent comprehension along with benefits of health outcomes of children [12]. Therefore, strategies focused primarily on responsive parenting might promote the growth of the child through family dyadic relationships [13].

\section{Description of the intervention}

Family-centered care (FCC) is described as a healthcare approach that involves planning, delivery, and evaluation centered on equally positive relationships among families, healthcare providers, and patients. FCC's values include family care, inclusive family engagement, interaction, empathy for and integrity of families, and knowledge transformation [14]. It has been implemented in developed and a few of the developing countries [15]. FCC can be practiced in various settings such as homes, clinics, hospitals, and communities. The central focus of the FCC is to train parents as a primary therapist and provide psychosocial support [14]. FCC has shown to be a promising approach for children ( $0-12$ years of age) by providing faster recovery, emotional/behavioral support to a child, parental satisfaction, and reduced cost due to hospitalization [16].

\section{How the intervention might work}

Approaches such as developmental care, Newborn Developmental Care and Assessment Program (NIDCAP), and Creating Opportunities for Parent Empowerment (COPE) have components of FCC, which are shown to be beneficial on medical and developmental outcomes of preterm infants [17-20]. Interaction between an infant and parent begins before infancy and is greater at childhood, with social-emotional experiences involving gestures, cries, smiles, and mutual gaze vocalizations, and continues throughout childhood [21]. A significant aspect affecting the growth of the child is the quality of interaction between an infant and parents. Modification of an infant's physical and emotional environment both in the NICU and post-hospitalization at home will have positive outcomes in their overall development $[22,23]$. The FCC has shown an increased knowledge, capacity, 
and competence of the parents to care for their infant or child [24]. The potential benefit of the FCC is that it is relatively low cost and has the focal involvement of parents for a long-term period. It has been shown to decrease the length of hospitalization, improve the wellbeing of preterm by allowing better allocation of resources, and enhance parent-infant bonding [24, 25]. In spite of benefits, FCC may make preterm infants vulnerable to infections transmitted through parents/family members or may catch hospital-acquired infections. However, a neonatal infection can be prevented with precautionary measures such as hand hygiene, proper handling techniques, clean clothing, and environmental hygiene [26].

\section{Why it is important to do this review}

In infants born very preterm, certain aspects of motor and neurobehavior function are frequently impaired relative to their counterparts born at term. FCC has been found to support the infant's care by enriching the environment, improving cognitive and physical growth, and further promoting early developmental resilience (right from the birth) [27, 28]. One of the reviews described that family-based interventions reduce preterm parental stress, and it has cost-effective benefits when practiced in the NICUs [25]. There was a significant improvement in weight gain, reduced hospital readmission, and positive parental outcomes (skills of infant care; parent satisfaction; reduced anxiety, stress, and depression) among the FCC intervention group when compared to the control group [13]. However, the review [13] did not address the effect of FCC on motor and neurobehavior development among infants. Other existing reviews were on developmental care intervention, and the intervention was delivered by health professionals alone and not by involving parents $[17,18]$. Additionally, these reviews were conducted on infants born at 37 or lesser weeks of gestation without subgroup of very preterm born infant. Hence, there is a need to explore the effects of FCC on motor and neurobehavior development of preterm infants.

Literature suggests that a supportive family environment, a positive bond between parent and infant, leads to improved neurodevelopmental outcomes even if the preterm infants are exposed to vulnerabilities such as neurological abnormality $[28,29]$. The studies have shown that negative and intrusive parenting leads to poor developmental outcomes in preterm children across childhood. On the contrary, warm, sensitive, and positive parenting might result in a protective effect on the preterm infant's development [30].

However, to the best of our knowledge, no systematic review has studied the impact of FCC interventions on the motor and neurobehavioral function in very preterm infants during both the NICU stay and the follow-up period. Furthermore, the various factors (mode of delivering the intervention, dosage, etc.) that might influence the success of FCC are not yet known. Hence, we would anticipate that the findings from this review will help inform clinicians, parents, and educators about the role of the FCC in promoting motor and neurobehavioral development in preterm infants. With this background, we have proposed two research questions: (1) What is the evidence of data available on the impact of FCC on very preterm infants when compared to standard care/interventions without involving a family on motor and neurobehavior development? (2) What are the factors that determine infant development due to FCC interventions?

We have described the relationship between the very preterm infant and FCC along with their possible outcomes through a conceptual framework (Fig. 1). In NICU, if there is limited parent-infant interaction, this might result in parental stress and poor bonding between them. This might further impact on delay or poor motor and neurobehavioral development of the infants and increases the level of parent anxiety and stress. On the other hand, involving parents or implementing the FCC right from the beginning in the NICU might create a well-nurturing environment and a positive parentinfant bonding due to the effect of various sensory experiences and activity-dependent brain activation. Further, this might accelerate the development of motor and neurobehavioral function along with the increased ability of learning and memory in preterm infants.

\section{Methods}

\section{Review registration and reporting}

The systematic review has been registered in PROSPERO (the International Prospective Register of Systematic Reviews) on August 26, 2020. We have adhered to PRISMA-P (Preferred Reporting Items for Systematic Review and Meta-analysis) guidelines to report this protocol (supplementary data file 1).

\section{Eligibility criteria \\ Types of studies}

Randomized controlled trials and quasi-randomized trials having at least two groups are eligible to be included. Non-randomized trials, observational studies, study protocols, editorials, reviews, conference abstracts, letters, and commentaries will be excluded.

\section{Type of participants}

Preterm infants born lesser than 32 weeks of gestation and their primary caregivers. Primary caregivers can be either mother, father, or grandparents. 


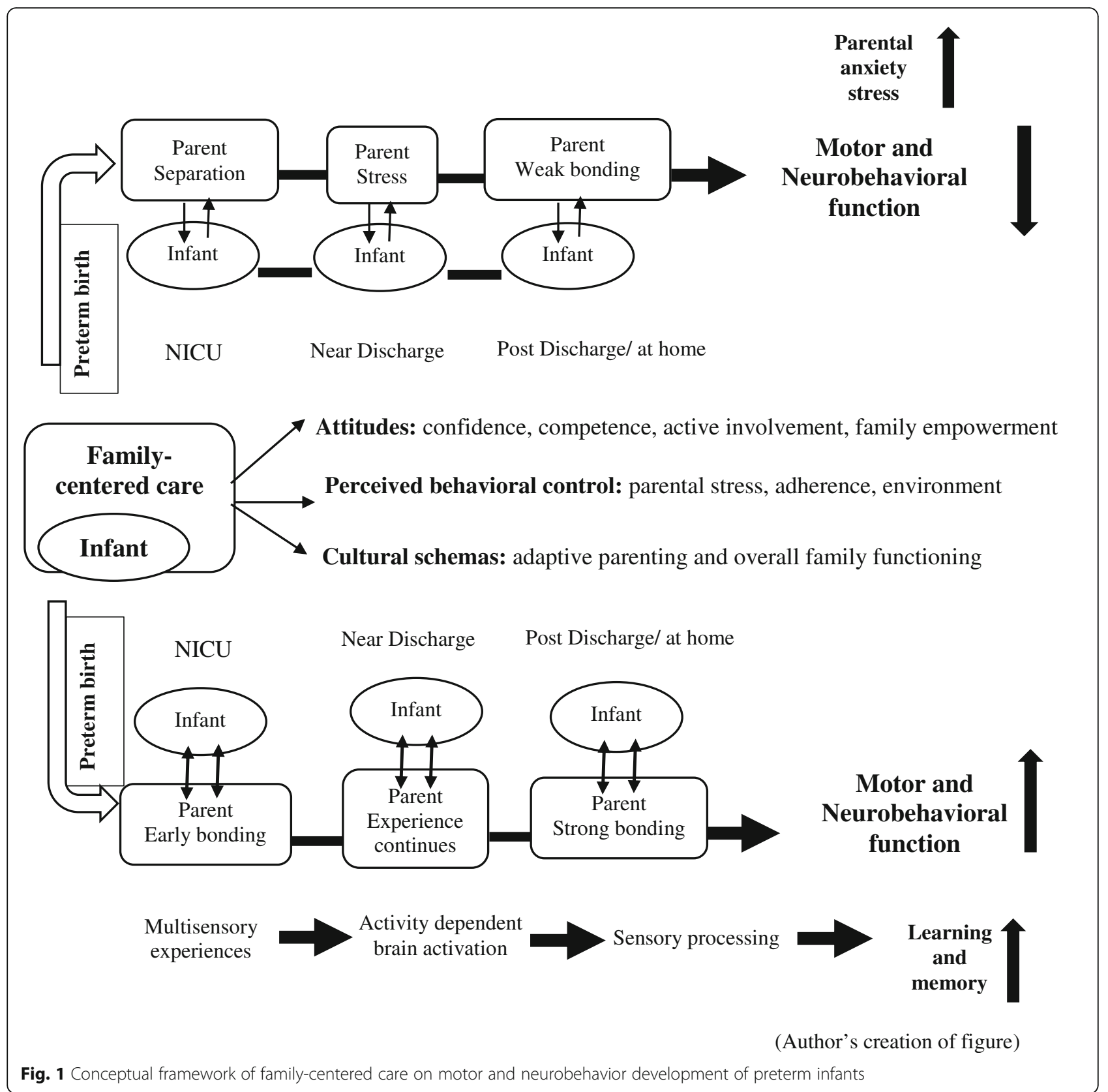

\section{Place of recruitment}

This review does not impose any restrictions on the setting. It can be in the NICU, hospital, and home or community.

\section{Time of recruitment and follow-up}

The studies should have recruited infants from birth in the NICU stay to 6 months, and followed up till 2 years of age.

\section{Intervention}

This review will include any FCC interventions involving the establishment of a collaborative relationship between the healthcare professional and the parent, mutually agreed-upon goal setting, and creating the home program by selecting therapeutic activities that focus on accomplishing family objectives, supporting the implementation of the program through home visits, parent education, and evaluating the outcomes. Interventions having at least two components of FCC will be included. The intervention will involve supervision and support from a clinician or professional such as a neonatologist, pediatrician, nurse, physiotherapist, occupational therapist, speech-language pathologists, and other rehabilitation team members. 


\section{Type of comparators}

The studies should have compared the FCC to the therapist's provided standard care interventions or usual care. Studies comparing a type of FCC versus another type of FCC will be included.

\section{Type of exposure}

Such as age, time of recruitment and follow-up, settings, FCC providers, intensity and frequency of intervention, parental behavior, responsivity, and parental satisfaction that may influence infant development.

\section{Types of outcome measures}

Primary outcomes We will include studies that measure motor and neurobehavioral function. Outcomes assessed at any time points but up to 2 years of age will be considered.

Motor functions may include quality of movement, gross and fine motor skills, developmental milestones, visual-spatial, visual-motor integration, balance, and coordination [6]. The tools used to measure motor functions could be Prechtl's General Movements Assessment (GMA), Test of Infant Motor Performance (TIMP), Alberta Infant Motor Scale (AIMS), Neuromotor Behavioral Assessment (NMBA), Hammersmith Infant Neurological Examination (HINE), Pediatric Evaluation of Disability Inventory (PEDI), Peabody Developmental Motor Scale (PDMS), and Bayley Scale of Infant and Toddler Development (BSID).

Neurobehavior is measured in terms of the sensory and autonomic nervous system; organization of state (calm, excited, irritable) and self-regulation (hand to mouth responses); language, attention, socio-emotional development; and executive function [7]. Neurobehavior could be measured using Assessment of Preterm Infants Behavior (APIB), Brazelton Neonatal Behavioral Assessment Scale (NBAS), Neurobehavioral Assessment of Preterm Infants (NAPI), and NICU Network Neurobehavioral Scale (NNNS).

Secondary outcomes are changes in parental behaviors or responsivity captured through videotaped interactions or observations and measured by any of the validated scales. Parental satisfaction will be measured by questionnaires and interviews. Factors such as age, time of recruitment and follow-up, settings, FCC providers, intensity, and frequency of intervention that might influence an infant's development will be considered for the review. We will also consider potential harms or risks of FCC intervention as reported by included studies, which may include, but not limited to, adverse events related to neonate, e.g., infections, and adverse events related to parent, e.g., anxiety.

\section{Search methods for identification of studies Electronic databases}

We will search PubMed, Cochrane Central, Scopus, Embase, PsycINFO (Ovid SP), CINAHL, and Web of Science. Articles that are written in English from January 2010 to August 15, 2020, will be included. The following keywords will be used: "family-centered care," "familycentric approach," "preterm infants," "motor development," and "neurobehavior development."

\section{Searching other resources}

We will search in ProQuest, ClinicalTrials.gov, EU Clinical Trials Register, metaRegister of Controlled Trials, and the World Health Organization (WHO) International Clinical Trials Registry Platform search portal. We will contact field experts and corresponding researchers of included studies to get relevant additional information. To find related studies, we shall search for reference lists and forward citations of included studies. Identified records will be exported to EndNote X7 for data management.

\section{Selection of studies}

Two reviewers will search and read the titles and abstracts of the listed sources individually and exclude any studies depending on the eligibility criteria. If disparity arises between the authors, it will be resolved by discussions and will be reviewed as to their full text. After obtaining the full text of the included abstracts, three review authors will independently rank these as "include" or "exclude." If necessary, we will address any differences in agreement, with a senior review author. We will record the reasons for excluding the articles. The proposed screening protocol for abstracts and full texts has been attached as supplementary data file 2 . Reference details, any knowledge accessible on current research, and record details of similar publication will be considered. An adapted PRISMA will be implemented (Fig. 2).

\section{Data extraction and management}

The extraction of general characteristics such as study identifiers, location of the study, participants, study selection criteria, and outcomes from the included studies will be carried out. The TIDier (Template for Intervention Description and Replication) checklist will be used to summarize the list of intervention characteristics and assist in the replicability of interventions and comparability between the studies [31]. Independently, two review authors will abstract the data from the articles included in the review using the data extraction templates. From each of the included studies, the following information will be extracted: study identification (title and authors), the country of study, year of publication, sample size, features of intervention, outcomes, 


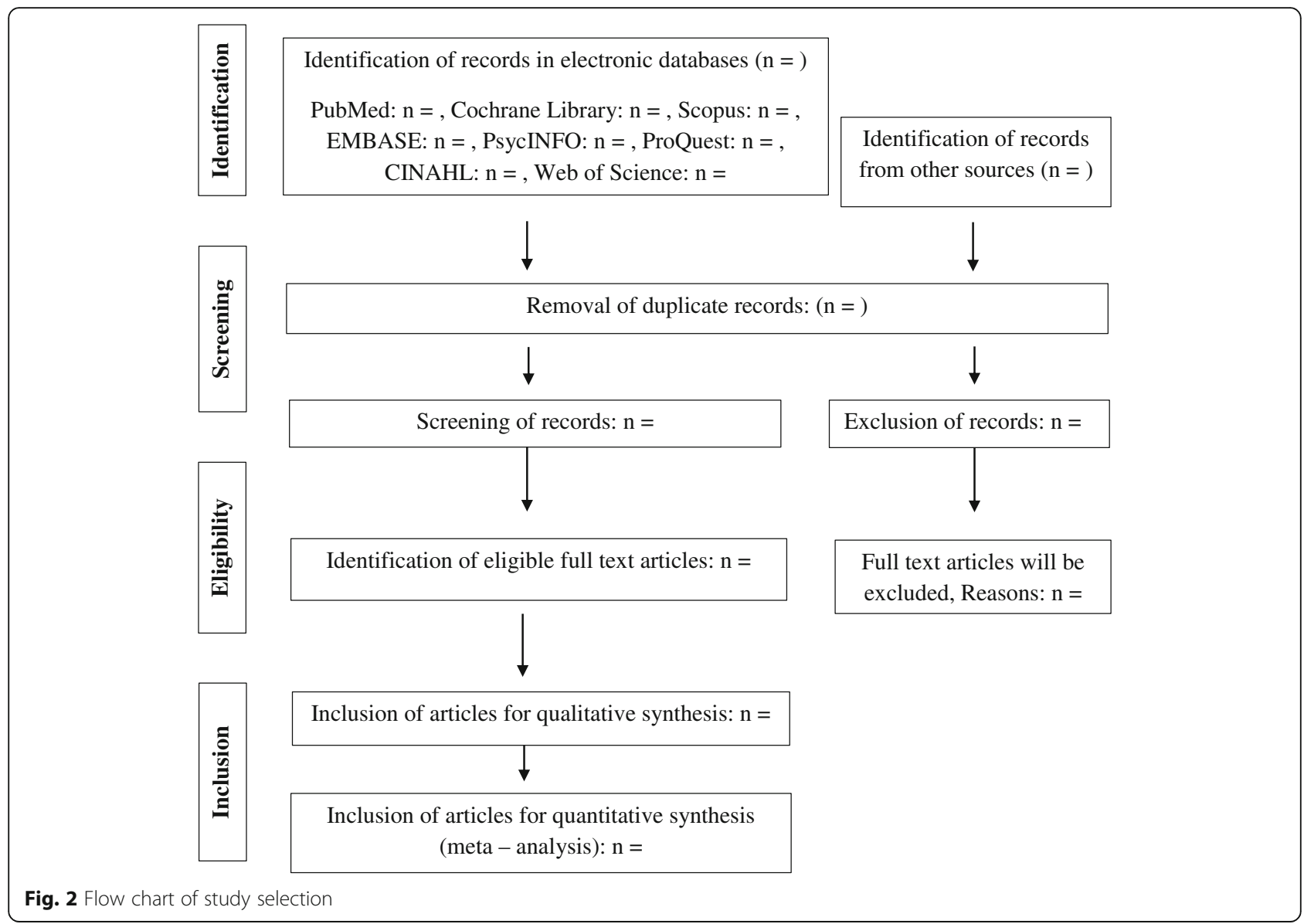

evaluation tools, results, and conclusions. In case of disagreement, we will discuss until a consensus, or with the help of the third reviewer, any disagreements will be resolved.

\section{Addressing redundant and related publications}

In the case of redundant papers, related records, or several primary research studies, we can optimize the data yield by gathering all the relevant details by utilizing the highly broad dataset collected through each of the documented papers. In correlation with our results, the publication which reports the longest follow-up will be considered as a priority.

\section{Risk of bias assessment among included studies}

The Revised Cochrane Risk of Bias Tool (RoB 2.0) would be employed to critically appraise the quality of the included articles. The RoB 2.0 tool assesses the randomization process, deviations from the intended protocol, measurement of the outcome, missing outcome data, and selective reporting. Two authors will appraise all the articles, independently. There should be agreement about the existence of certain inconsistencies.
The risk will be categorized into low risk, high risk, and some concern [32].

\section{Addressing the missing data}

From the authors, missing information would be collected, and significant empirical data such as screened, randomized, intention-to-treat, as-treated, and perprotocol population will be closely analyzed. If authors will not respond within 15 days of the last communication (email), the study would be excluded from the review. We will critically appraise the concerns related to the missing data and imputation methods, e.g., last observation carried forward (LOCF). The attrition rates, e.g., drop-outs, losses to follow-up, and withdrawals, will be investigated.

\section{Data synthesis}

The outcomes will be presented as the mean differences (MDs)/standardized mean differences (SMDs) with 95\% confidence intervals (CI) unless otherwise stated for continuous variable. For dichotomous data, the effect estimate is risk ratios (RRs) with 95\% CI. If there is strong evidence of homogeneous effects through findings, using a model of random effects we will mainly sum up a low 
probability of bias results. The meta-analysis of random effects will be viewed with due consideration of the full range of outcomes, preferably by providing an interval of predictions. In each study, the predicted range for the true treatment effect will be specified using the prediction interval. The quality of evidence of the included studies in the review will be assessed using the Grading of Recommendations Assessment, Development, and Evaluation (GRADE) system. This system grades quality of evidence at 4 levels: high (4), moderate (3), low (2), and very low (1) [33].

\section{Subgroup analyses and investigation of heterogeneity}

In the meta-analysis, we will not record the findings of the sample as the pooled effect estimate if there is an event of major scientific, analytical, or empirical variability. Using a standard Chi-square test (significance level of $\alpha=0.1$ ), we will recognize the heterogeneity. Using the $I^{2}$ statistics, inconsistency across studies will be quantified. It is considered to be a high level of inconsistency if an $I^{2}$ statistic is $75 \%$ or more. If there is significant variability, we will aim to identify potential explanations for this by analyzing the features of the specific study and other subgroups. Subgroup analyses of factors such as gender, age, country, type, and mode of intervention and outcome measures (or different time points) will be carried out to explore the interaction between them.

\section{Sensitivity analysis}

To understand how the following factors influence the effect sizes, we will perform sensitivity analyses. The factors include the risk of bias of included studies and large and long trials to understand the extent to which they influence the results. Different effect size measurements such as risk ratio and odds ratio along with various statistical models such as fixed effects and random effects models will be used to test the robustness of the results.

\section{Narrative synthesis}

When it is not possible to conduct the meta-analysis because of significant statistical, clinical, or methodological heterogeneity, a narrative synthesis will be done. Studies would be narratively defined focusing on the intervention and outcomes. The subsequent results would be summarized using the tables and figures.

\section{Discussion}

Through FCC, the health professionals guide the programs and work in partnership with the parents to support and guide the infant's motor and neurobehavior development to enhance their capabilities. Providing care to the preterm infants through a family-centered approach may improve the overall infant development and in turn reduces the burden of the caregivers and enhances their capacities. The findings of this review will provide an understanding of the effectiveness of FCC components and their benefits on very preterm infants and thereby help policymakers and health professionals to adopt evidence-based decision making and practice of FCC. This systematic review will guide the clinicians on the feasibility of practicing FCC that might support and promote the integration of parents into various rehabilitation settings.

\section{Strengths and limitations}

To the best of our knowledge, this systematic review is the first to address the effect of the FCC on improving motor and neurobehavioral outcomes in preterm infants and the factors influencing infant development. We will undertake a comprehensive search in various databases to identify the studies; however, due to resource constraint, studies published in English will be considered.

\section{Supplementary Information}

The online version contains supplementary material available at https://doi. org/10.1186/s13643-021-01612-w.

Additional file 1: Supplementary data file 1. PRISMA-P checklist.

Additional file 2: Supplementary data file 2. Proposed screening protocol for abstracts and full texts.

\section{Abbreviations}

FCC: Family-centered care; NICU: Neonatal intensive care unit; PROSPERO: The International Prospective Register of Systematic Reviews; NIDCAP: Newborn Developmental Care and Assessment Program; COPE: Creating Opportunities for Parent Empowerment; PRISMA-P: Preferred Reporting Items for Systematic Review and Meta-analysis; GMA: General Movements Assessment; TIMP: Test of Infant Motor Performance; AIMS: Alberta Infant Motor Scale; NMBA: Neuromotor behavioral assessment; HINE: Hammersmith Infant Neurological Examination; PEDI: Pediatric Evaluation of Disability Inventory; PDMS: Peabody Developmental Motor Scale; BSID: Bayley Scale of Infant and Toddler Development;

APIB: Assessment of Preterm Infants Behavior; NBAS: Neonatal Behavioral Assessment Scale; NAPI: Neurobehavioral Assessment of Preterm Infants; NNNS: NICU Network Neurobehavioral Scale; TIDier checklist: Template for Intervention Description and Replication; ROB 2.0 Tool: The Revised Cochrane Risk of Bias; LOCF: Last Observation Carried Forward; WHO: World Health Organization; MD: Mean difference; SMD: Standardized mean difference; OR: Odds ratio; RR: Risk ratio; Cl: Confidence interval; GRADE: Grading of Recommendations Assessment, Development and Evaluation

\section{Acknowledgements}

The authors would like to acknowledge Manipal Academy of Higher Education, Manipal, for technical support.

Authors' contributions

BK and MK conceived the topic. SSP, BK, AS, and MK designed the protocol. $B K$ coordinated with the team and timeline to finalize the protocol. BK, SR, MK, AS, and SSP designed the search strategies. MK, BK, and SSP wrote the protocol. All authors read, provided general advice, and approved the final version of the protocol. BK is the guarantor of the review.

Funding

The authors declare no source of funding. 


\section{Availability of data and materials}

The data acquisition for this systematic review has not yet started. We plan to conduct the search in mid-August. Subsequently, dataset generated through this systematic review can be requested from the corresponding author.

\section{Ethics approval and consent to participate}

Ethical approval is not applicable as this is a systematic review protocol; we will not involve the human population directly.

\section{Consent for publication}

Not applicable as the manuscript does not contain data from any person.

\section{Competing interests}

The authors declare no competing interests.

\section{Author details}

'Department of Physiotherapy, Manipal College of Health Professions, Manipal Academy of Higher Education, Manipal 576104, India. ${ }^{2}$ Department of Physiotherapy, University of Melbourne, Melbourne, Australia. ${ }^{3}$ Public Health Evidence South Asia, Prasanna School of Public Health, Manipal Academy of Higher Education, Manipal, India.

\section{Received: 24 August 2020 Accepted: 9 February 2021}

\section{Published online: 18 February 2021}

\section{References}

1. World Health Organization. Preterm birth. Geneva: WHO; 2018. Accessed 14 July 2020. Available from: https://www.who.int/news-room/fact-sheets/deta il/preterm-birth

2. Blencowe $\mathrm{H}$, Cousens $\mathrm{S}$, Chou D, et al. Born too soon: the global epidemiology of 15 million preterm births. Reprod Health. 2013;10(Suppl 1): S2.

3. Centers for Disease Control and Prevention. User guide to the 2016 period linked birth/infant death public use file. Available at: https://ftp.cdc.gov/ pub/Health_Statistics/NCHS/Dataset_Documentation/DVS/periodlinked/ LinkPE16Guide.pdf. Accessed 13 Jan 2021.

4. George JM, Fiori S, Fripp J, Pannek K, Guzzetta A, David M, et al. Relationship between very early brain structure and neuromotor, neurological and neurobehavioral function in infants born $<31$ weeks gestational age. Early Hum Dev. 2018;117:74-82.

5. van't Hooft J, van der Lee JH, Opmeer BC, Aarnoudse-Moens CS, Leenders AG, Mol BW, et al. Predicting developmental outcomes in premature infants by term equivalent MRI: systematic review and meta-analysis. Syst Rev. 2015;4:71

6. Adolph KE, Franchak JM. The development of motor behavior. Wiley Interdiscip Rev Cogn Sci. 2017;8(1-2):10.

7. Craciunoiu $\mathrm{O}$, Holsti L. A systematic review of the predictive validity of neurobehavioral assessments during the preterm period. Phys Occup Ther Pediatr. 2017:37:292-307.

8. Kara ÖK, Şahin S, Kara K, Arslan M. Neuromotor, and sensory development in preterm infants: a prospective study. Turk Pediatri Ars. 2020;55(1):46-53.

9. Cabral TI, Pereira da Silva LG, Tudella E, Simões Martinez CM. Motor development and sensory processing: a comparative study between preterm and term infants. Res Dev Disabil. 2015:36C:102-7.

10. Bradley RH, Corwyn RF, McAdoo HP, Coll CG. The home environments of children in the United States part I: variations by age, ethnicity, and poverty status. Child Dev. 2001;72(6):1844-67.

11. Neri E, Genova F, Monti F, Trombini E, Biasini A, Stella M, et al. Developmental dimensions in preterm infants during the 1st year of life: the influence of severity of prematurity and maternal generalized anxiety. Front Psychol. 2020;11:455

12. Curran JA, Gallant AJ, Zemek R, Newton AS, Jabbour M, Chorney J, et al. Discharge communication practices in pediatric emergency care: a systematic review and narrative synthesis. Syst Rev. 2019;8(1):83.

13. Ding $X$, Zhu L, Zhang R, Wang L, Wang TT, Latour JM. Effects of familycentered care interventions on preterm infants and parents in neonatal intensive care units: a systematic review and meta-analysis of randomized controlled trials. Aust Crit Care. 2019;32(1):63-75.

14. Committee on Hospital Care, American Academy of Pediatrics. Family centered care and the pediatrician's role. Pediatrics. 2003;112(3 Pt 1):691-7.
15. Sarin E, Maria A. Acceptability of a family-centered newborn care mode among providers and receivers of care in a public health setting: a qualitative study from India. BMC Health Serv Res. 2019;19(1):184.

16. Shields L, Zhou H, Pratt J, Taylor M, Hunter J, Pascoe E. Family-centred care for hospitalized children aged 0-12 years. Cochrane Database Syst Rev. 2012; 10:CD004811.

17. Soleimani F, Azari N, Ghiasvand H, Shahrokhi A, Rahmani N, Fatollahierad S. Do NICU developmental care improve cognitive and motor outcomes for preterm infants? A systematic review and meta-analysis. BMC Pediatr. 2020; 20(1):67.

18. Legendre V, Burtner PA, Martinez KL, Crowe TK. The evolving practice of developmental care in the neonatal unit: a systematic review. Phys Occup Ther Pediatr. 2011;31(3):315-38.

19. Mianaei SJ, Karahroudy FA, Rassouli M, Tafreshi MZ. The effect of Creating Opportunities for Parent Empowerment program on maternal stress, anxiety, and participation in NICU wards in Iran. Iran J Nurs Midwifery Res. 2014;19(1):94-100.

20. Spittle A, Treyvaud K. The role of early developmental intervention to influence neurobehavioral outcomes of children born preterm. Semin Perinatol. 2016;40(8):542-8.

21. Franck LS, O'Brien K. The evolution of family-centered care: from supporting parent-delivered interventions to a model of family integrated care. Birth Defects Res. 2019;111(15):1044-59.

22. Kokorelias KM, Gignac MAM, Naglie G, Cameron Jl. Towards a universal model of family-centered care: a scoping review. BMC Health Serv Res. 2019;19(1):564.

23. Yu YT, Hsieh WS, Hsu CH, et al. Family-centered care improved neonatal medical and neurobehavioral outcomes in preterm infants: randomized controlled trial. Phys Ther. 2017;97(12):1158-68.

24. Verma A, Maria A, Pandey RM, Hans C, Verma A, Sherwani F. Familycentered care to complement care of sick newborns: a randomized controlled trial. Indian Pediatr. 2017;54(6):455-9.

25. Welch MG, Myers MM. Advances in family-based interventions in the neonatal ICU. Curr Opin Pediatr. 2016;28(2):163-9.

26. Coffin SE, Zaoutis TE. Healthcare-associated infections in the nursery. In: Remington JS, Klein JO, Wilson CB, Nizet V, Maldonado YA, editors. Infectious Diseases of the Fetus and Newborn. 7th ed. Philadelphia: W. B. Saunders; 2011. p. 1126-43.

27. McEwen BS. Early life influences on life-long patterns of behavior and health. Ment Retard Dev Disabil Res Rev. 2003;9(3):149-54.

28. Treyvaud K, Doyle LW, Lee KJ, Ure A, Inder TE, Hunt RW, et al. Parenting behavior at 2 years predicts school-age performance at 7 years in very preterm children. J Child Psychol Psychiatry. 2016;57(7):814-21.

29. Treyvaud K, Inder TE, Lee KJ, Northam EA, Doyle LW, Anderson PJ. Can the home environment promote resilience for children born very preterm in the context of social and medical risk? J Exp Child Psychol. 2012;112(3):32637.

30. Treyvaud K, Anderson VA, Howard K, Bear M, Hunt RW, Doyle LW, et al. Parenting behavior is associated with the early neurobehavioral development of very preterm children. Pediatrics. 2009:123(2):555-61.

31. Hoffmann T, Glasziou P, Boutron I, Milne R, Perera R, Moher D, et al. Better reporting of interventions: template for intervention description and replication (TIDieR) checklist and guide. BMJ. 2014;348:g1687.

32. Sterne JAC, Savović J, Page MJ, Elbers RG, Blencowe NS, Boutron I, et al. RoB 2: a revised tool for assessing the risk of bias in randomized trials. BMJ. 2019;366:14898.

33. Guyatt GH, Oxman AD, Akl E, Kunz R, Vist G, Brozek J, et al. GRADE guidelines 1. Introduction-GRADE evidence profiles and summary of findings tables. J Clin Epidemiol. 2011;64:383-94.

\section{Publisher's Note}

Springer Nature remains neutral with regard to jurisdictional claims in published maps and institutional affiliations. 